Chronic Obstructive Pulmonary Diseases:

Journal of the COPD Foundation

\author{
Original Research
}

\title{
The Effects of a Comprehensive Care Management Program on Readmission Rates After Acute Exacerbation of COPD at a Community-Based Academic Hospital
}

\author{
Glenda Euceda, $\mathrm{MD}^{1}$ Wing-Tai Kong, $\mathrm{MD}^{1}$ Amber Kapoor, $\mathrm{MPH}^{2}$ Patricia Dilauro, RN, RRT ${ }^{3}$ \\ Rahila Ogunnaike, $\mathrm{MD}^{1}$ John Chronakos, $\mathrm{MD}^{3}$
}

\begin{abstract}
Acute exacerbation of chronic obstructive pulmonary disease (AECOPD) is one of the leading causes of hospitalization in the United States. Prior investigations suggest clinical and physiological parameters are important determinants for AECOPD readmissions. Strategies aimed at addressing these factors have not resulted in a major reduction of readmissions. We compared patients readmitted after an index AECOPD admission with non-readmitted patients. Patients' age, gender, body mass index, comorbidities (obstructive sleep apnea, chronic hypercapnia, congestive heart failure, lung cancer, pulmonary arterial hypertension, pneumonia, interstitial lung disease, atrial fibrillation, musculoskeletal disorders, cognitive disorders, and anxiety disorders), substance abuse and smoking status were assessed. Some 272 patients were included: 20 patients were readmitted within 30 days of their index hospitalization; 252 patients were not readmitted within 30 days of their index admission. Readmitted patients were significantly more likely to have pneumonia than non-readmitted patients $(30.0 \%$ versus $13.1 \%, p<0.05)$. No statistically significant difference was seen with respect to other clinical comorbidities. Patients readmitted within 30 days were significantly more likely than non-readmitted patients to have safety issues at home $(80.0 \%$ versus. $39.3 \%, p<0.001)$, anxiety $(60.0 \%$ versus $29.8 \%, p<0.01$ ), and lack of transportation (35.0\% versus $15.5 \%, p<0.05)$. Implementation of a comprehensive care management program (CCMP) was associated with a reduction in readmissions from $21.5 \%$ to $13.6 \%$ $(p<0.01,95 \%$ confidence interval [CI] 2.08-12.45). A CCMP can reduce readmissions through attention to social variables, optimization of in-hospital care, improved coordination of pre- and post-discharge, a system to better identify problems after discharge, and an office setup that accommodates same-day visits.
\end{abstract}

\footnotetext{
Abbreviations: acute exacerbation of chronic obstructive pulmonary disease, AECOPD; chronic obstructive pulmonary disease, COPD; Centers for Medicare and Medicaid Services, CMS; comprehensive care management program, CCMP; Global initiative for chronic Obstructive Lung Disease, GOLD; forced expiratory volume in 1 second, FEV $\mathbf{1}$; forced vital capacity, FVC; body mass index, BMI; Home Safety Self-Assessment Tool, HSSAT; confidence interval, CI

Funding Support: The study was supported by the Western Connecticut Health Network Foundation.

Date of Acceptance: February 21, 2018

Citation: Euceda G, Kong W-T, Kapoor A, Dilauro P, Ogunnaike R, Chronakos J. The effects of a comprehensive care management program on readmission rates after acute exacerbation of COPD at a community-based academic hospital. Chronic Obstr Pulm Dis. 2018;5(3):185192. doi: https://doi.org/10.15326/jcopdf.5.3.2017.0177
}

1 Department of Internal Medicine, Danbury Hospital, Western Connecticut Health Network, Danbury

2 Department of Research \& Innovation, Danbury Hospital, Western

Connecticut Health Network, Danbury
3 Section of Pulmonary Medicine, Department of Internal Medicine, Danbury Hospital, Western Connecticut Health Network, Danbury 


\section{Address correspondence to:}

John Chronakos, MD

33 Germantown Road

Danbury, CT 06810

E-mail: john.chronakos@gmail.com

Phone: (203-)739-6805

\section{Keywords:}

population health; rehospitalization prevention; risk factors for rehospitalization

\section{Introduction}

Acute exacerbation of chronic obstructive pulmonary disorder (AECOPD) is one of the leading causes of hospitalization in the United States. ${ }^{1}$ The Agency for Healthcare Research and Quality estimates that 1 out of every 5 patients over the age of 40 who have been hospitalized in the past decade carries a diagnosis of chronic obstructive pulmonary disease (COPD). ${ }^{2}$ Given its prevalence in hospitalized patients, COPD and its acute exacerbation pose a heavy financial burden in health care. ${ }^{1}$ As a result, reducing the frequency of hospitalizations for patients with AECOPD has become a high priority for health care organizations and government agencies, including the U.S. Centers for Medicare and Medicaid Services (CMS). ${ }^{3}$ In 2013, CMS identified the readmission of patients hospitalized for COPD as a measure for hospital performance. ${ }^{3}$ Hospitals across the country are assessed on their ability to reduce 30-day all-cause unplanned readmissions after an initial admission for a COPD exacerbation. Furthermore, as CMS has tied readmission rates to hospital payments, reducing readmission rates for COPD exacerbation has become a major concern for hospitals.

Several previous studies have shown that many risk factors are associated with readmission in patients with AECOPD, including poor lung function, low oxygen saturation on pulse oximetry, history of cor pulmonale, muscle weakness, decreased activity levels, other medical comorbidities, and poor medication reconciliation during hospitalizations. ${ }^{4}$ In 2013, Park et al found that the prevalence of frailty was $58 \%$ higher in patients with COPD aged 55 years or older compared to that of the general population, as assessed by a frailty tool which included weight loss, difficulty walking, weakness, cognitive impairment and vision and hearing impairment. ${ }^{5}$ In 2 cross-sectional studies, Anecchino et al and Holguin et al demonstrated that $68 \%$ of patients admitted with AECOPD have at least 1 comorbidity, $16 \%$ have 2 or more, and $30 \%$ of patients have 4 or more comorbidities. ${ }^{6,7}$ In addition, according to a study by Shah et al, only $27.6 \%$ of all readmissions of COPD carry the same diagnosis as the index admission, ${ }^{8}$ suggesting that clinical comorbidities might play an important role in COPD readmissions. The increased prevalence of frailty and other comorbidities place patients with COPD at higher than average readmission risk.

While prior investigations have suggested that clinical and physiological parameters might be important determinants for COPD readmissions, specific strategies aimed at decreasing readmissions by addressing the factors above have not been formally investigated. ${ }^{9}$ This suggests that other risk factors that have not yet been identified might also play an important role. In 2011, Coventry, Gemmell and Todd showed that depression and lack of home ownership were both associated with an increased frequency of readmissions for patients with AECOPD. ${ }^{4}$ Although there is conflicting evidence, there is some support for the effect differing levels of social support and socioeconomic factors has on AECOPD readmission. Living with a partner was identified as a protective factor for males with COPD, while lower socioeconomic status, inequalities in education, and income were associated with a 3 -fold increase in risk for readmission. ${ }^{4,10}$ These findings imply that psychosocial risk factors may also be drivers among patients who are readmitted.

Despite the studies mentioned above, there have not been well accepted management modalities and guidelines that address psychosocial risk factors for the prevention of COPD readmissions. Furthermore, a patient's ability to follow discharge instructions or take medications as prescribed at home is often poorly assessed, and optimal transition of care to a primary care provider remains challenging. ${ }^{9}$ As COPD exacerbation causes increased hospitalizations and poses a heavy financial burden to society, there is a great interest in decreasing readmissions for COPD exacerbation. One approach to reduce readmissions and control costs being utilized is a comprehensive care management program (CCMP). A Cochrane review of 26 studies in 2014 demonstrated a reduction in respiratory-related readmissions and hospital days when a CCMP was utilized. ${ }^{11}$ Other studies, however, have shown that such programs have had varied success rates. ${ }^{12-16}$ 
This study aims to investigate both clinical and psychosocial risk factors associated with readmissions for COPD exacerbation among patients of an academic community hospital which implemented a CCMP. In doing so, we seek to better understand and improve strategies that can further reduce readmissions for patients with COPD exacerbation.

\section{Methods}

\section{Study Design}

A COPD readmission reduction initiative was implemented in September 2014 at Danbury Hospital, a community teaching hospital in Connecticut. The program was developed to address readmission issues as part of an overall quality improvement program. The program set up teams of inpatient and outpatient care navigators, supervised by collaborating physicians, whose job was to facilitate the transition from hospital to home. These navigators concentrated on reviewing discharge medication reconciliation, addressing anxiety issues, assessing financial difficulties related to buying medication, teaching correct inhaler use via the teach-back method, and helping patients better understand discharge instructions. Patients were called within 48 hours of discharge by the care navigator and at least weekly throughout the following month, in coordination with a prompt hospital discharge followup with a pulmonologist within 5 days.

This study is a retrospective analysis of patients admitted to Danbury Hospital with a diagnosis of AECOPD between September 2014 and September 2015. The study was designed to assess the risk factors associated with patients who are readmitted for any reason within 30 days of a COPD exacerbation. The study protocol was reviewed and approved by the Biomedical Research Alliance of New York Institutional Review Board who determined that informed consent could be waived given the nature of the study. All guidelines of the Declaration of Helsinki were followed.

\section{Patient Population}

With the implementation of the COPD readmission reduction initiative, patients admitted to Danbury Hospital with a diagnosis of COPD exacerbation were evaluated by a pulmonologist. The diagnosis of COPD was made according to the Global initiative for chronic Obstructive Lung Disease standard, ${ }^{17}$ defined as a ratio of forced expiratory volume in 1 second ( $F E V_{1}$ ) to forced vital capacity (FVC) of $<0.7$, after bronchodilator use. In the absence of pulmonary function tests, presumptive diagnoses were made by the consulting pulmonologist based on risk factors such as a smoking history of more than 20 pack years, the presence of chronic respiratory symptoms, or other corroborating evidence such as the presence of emphysematous changes on a computerized tomography scan or chest radiograph. Exacerbation of COPD was defined as increased cough, increased sputum, change in sputum color, or increased dyspnea in a patient with COPD. All patients with a diagnosis of AECOPD were included in the study, save for a small number (fewer than $10 \%$ of patients) who were cared for by a private communitybased pulmonary group or who expired on the index admission or were on an end-of-life pathway such as being discharged to hospice.

\section{Data Collection}

Data were collected and entered by an outpatientbased COPD care navigator. A detailed description of variables is listed in Table 1. Patients' age, gender, and body mass index (BMI) were collected. BMI was categorized as underweight $(\mathrm{BMI}<18.5)$, normal weight $(18.5 \leq \mathrm{BMI}<25)$, overweight $(25 \leq \mathrm{BMI}<30)$, and obese $(\mathrm{BMI} \geq 30) . \mathrm{FEV}_{1}$ was collected as a marker of COPD severity. Clinical comorbidities, including history of obstructive sleep apnea, chronic hypercapnia, congestive heart failure, lung cancer, pulmonary arterial hypertension, pneumonia, interstitial lung disease, atrial fibrillation, presence of musculoskeletal disorders, cognitive disorders, anxiety disorders, substance abuse, and smoking status were also assessed. Smoking status was categorized as current smoker, former smoker who quit within 3 months of index admission, former smoker who quit more than 3 months prior to index admission, and lifetime nonsmoker. Psychosocial risk factors including anxiety, home safety, and access to transportation were also evaluated. Anxiety was defined as diagnosis of anxiety disorder in the past, or current use of anti-anxiety medication such as a benzodiazepine. Medication non-adherence was defined as history of medication non-compliance, inability to comply with medication regimen, and/or inability to perform teach-back. The home safety assessment was evaluated by the COPD care navigator, in accordance with the Home Safety Self-Assessment Tool (HSSAT) by the International 


\section{Table 1. Detailed Definitions of Socioeconomic Variables}

\begin{tabular}{ll}
\hline Substance Abuse & Definition \\
& $\begin{array}{l}\text { Recent use of illegal drugs (including marijuana, cocaine, stimulants, or opiates); evidence of a } \\
\text { substance abuse disorder, physical dependence, or addiction; unhealthy alcohol use. }\end{array}$ \\
\hline Safety at Home & $\begin{array}{l}\text { Living alone, lack of caregiver in town, homelessness, unsafe home environments documented by care } \\
\text { coordinator. }\end{array}$ \\
\hline Anxiety & Diagnosis of anxiety disorder in the past, current use of anxiolytics including benzodiazepine. \\
\hline $\begin{array}{l}\text { Medication } \\
\text { Non-adherence }\end{array}$ & $\begin{array}{l}\text { History of medication non-compliance documented from previous EMR, inability to comply with } \\
\text { medication regimen and inability to perform teach-back during assessment of home and/or office visits. }\end{array}$ \\
\hline $\begin{array}{l}\text { Lack of } \\
\text { Transportation }\end{array}$ & $\begin{array}{l}\text { Lack of access to personal vehicles, or easily available transportation to outpatient appointments or } \\
\text { pharmacy. }\end{array}$ \\
\hline
\end{tabular}

EMR=electronic medical record

Classification for Patient Safety. ${ }^{18}$ Transportation issues included not having access to personal vehicles or other easily available transportation to outpatient appointments or pharmacies (like access-a-ride services).

\section{Statistical Analysis}

A two-tailed t-test was used to compare continuous variables; Chi-Square analyses were used for risk factor calculation among categorical variables. Twotailed Fisher's exact test was used in cases of expected small cell size and 95\% confidence intervals (CI) are reported for continuous variables; odds ratios and $95 \%$ CI are reported for categorical variables. Statistical significance was defined as $p<0.05$. The 30-day readmission rate was defined according to the Agency for Healthcare Research and Quality as the number of stays with at least 1 subsequent hospital stay for any reason within 30 days divided by the total number of hospital stays between September 2014 and September 2015. JMP version 11.1.1 (SAS Institute, Inc., Cary, North Carolina) was used to perform all statistical analyses.

\section{Results}

During the study period of September 2014 through September 2015, there were 366 admissions for COPD exacerbation among 291 unique patients. Of these, 19 patients passed away during index admission, were discharged to hospice, or were lost to follow-up by the COPD initiative program and were excluded from this study. Of the 272 patients included in this study, 20 patients were readmitted within 30 days of their index hospitalization; 252 patients were not readmitted within 30 days of their index hospitalization. The demographic characteristics of patients are summarized in Table 2. The mean age was 74.3 years (SD 10.5 years) among those who were readmitted and 73.1 years (SD 12.5 years) among those who had a single admission. There were no statistically significant differences between those who were readmitted and those who were not with respect to age $(p=0.66)$, smoking status $(p=0.46)$, gender, $(p=0.91)$ or BMI $(p=0.49)$. Mean FEV 1 also did not differ significantly between the groups ( $p=0.43$ ).

Clinical comorbidities are summarized in Table 2. Patients who were readmitted were significantly more likely to have pneumonia at their index admission than those who were not readmitted (30.0\% versus $13.1 \%$, $p<0.05)$. No statistically significant difference was seen between the 2 groups with respect to any other clinical comorbidities. Patients who were readmitted within 30 days were significantly more likely than those who were not readmitted to have safety issues at home $(80.0 \%$ versus $39.3 \%, p<0.001)$, anxiety $(60.0 \%$ versus $29.8 \%, p<0.01)$ and lack of transportation (35.0\% versus $15.5 \%, p<0.05$ ) (Table 3 ).

Monthly hospital-wide 30-day readmission rates for patients with COPD are presented in Figure 1. The annual readmission rate decreased from $21.5 \%$ in the one-year period prior to CCMP implementation to $13.6 \%$ in the one-year period post-implementation $(p<0.01$, CI 2.08-12.45).

\section{Discussion}

In this retrospective study, we compared patients who had been readmitted to Danbury Hospital after an index AECOPD admission with those who were not readmitted from September 2014 to September 2015. We hypothesized that patients who were readmitted following an AECOPD were more likely to have 


\section{Table 2. Demographic Information and Clinical Comorbidities}

\begin{tabular}{|c|c|c|c|c|}
\hline & $\begin{array}{c}\text { Readmitted } \\
\text { Within } 30 \text { Days } \\
\text { of Index Admission }\end{array}$ & $\begin{array}{c}\text { Not Readmitted } \\
\text { Within } 30 \text { Days } \\
\text { of Index Admission }\end{array}$ & $p$-value & $\begin{array}{l}\text { Odds Ratio } \\
(95 \% \mathrm{Cl})\end{array}$ \\
\hline Mean Age, years (SD) & $74.3(10.5)$ & $73.1(12.5)$ & 0.66 & - \\
\hline Male (\%) & $9(45.0)$ & $110(43.7)$ & 0.91 & $1.06(0.42-2.64)$ \\
\hline \multicolumn{3}{|l|}{ Smoking (\%) } & \multirow[t]{5}{*}{0.46} & \multirow[t]{5}{*}{-} \\
\hline Never Smoker & $1(5.0)$ & $11(4.4)$ & & \\
\hline Quit Over 3 Months & $11(55.0)$ & $136(54.0)$ & & \\
\hline Quit Within 3 Months & 0 & $18(7.1)$ & & \\
\hline Current Smoker & $8(40.0)$ & $83(32.9)$ & & \\
\hline \multicolumn{3}{|l|}{ Body Mass Index $(\%)$} & \multirow[t]{5}{*}{0.49} & \multirow[t]{5}{*}{-} \\
\hline Underweight & $3(15.0)$ & $22(8.7)$ & & \\
\hline Healthy & $5(25.0)$ & $98(38.9)$ & & \\
\hline Overweight & $7(35.0)$ & $64(25.4)$ & & \\
\hline Obese & $5(25.0)$ & $68(27.0)$ & & \\
\hline Mean FEV $_{1}$, liters per second (SD) & $1.18(0.47)$ & $1.28(0.55)$ & 0.43 & - \\
\hline Chronic Hypercapnia (\%) & $6(30.0)$ & $40(15.9)$ & 0.12 & $2.27(0.82-6.26)$ \\
\hline Obstructive Sleep Apnea (\%) & $3(15.0)$ & $28(11.1)$ & 0.49 & $1.41(0.39-5.12)$ \\
\hline Congestive Heart Failure (\%) & $2(10.0)$ & $52(20.6)$ & 0.38 & $0.43(0.10-1.90)$ \\
\hline Atrial Fibrillation $(\%)$ & $3(15.0)$ & $35(13.9)$ & 0.75 & $1.09(0.30-3.93)$ \\
\hline Pulmonary Arterial Hypertension (\%) & $2(10.0)$ & $16(6.4)$ & 0.63 & $1.64(0.35-7.69)$ \\
\hline Musculoskeletal Disorder (\%) & $8(40.0)$ & $71(28.2)$ & 0.28 & $1.70(0.67-4.33)$ \\
\hline Cognitive Disorder $(\%)$ & $3(15.0)$ & $34(13.5)$ & 0.74 & $1.13(0.31-4.07)$ \\
\hline Lung Cancer $(\%)$ & 0 & $12(4.8)$ & 1 & - \\
\hline Pneumonia (\%) & $6(30.0)$ & $33(13.1)$ & 0.049 & $2.84(1.02-7.91)$ \\
\hline Interstitial Lung Disease (\%) & $1(5.0)$ & $2(0.8)$ & 0.21 & $6.58(0.57-75.89)$ \\
\hline
\end{tabular}

$\mathrm{CI}=$ confidence interval; $\mathrm{SD}=$ standard deviation; $\mathrm{FEV}_{1}=$ forced expiratory volume in 1 second

\section{Table 3. Social Issues}

\begin{tabular}{|c|c|c|c|c|}
\hline & $\begin{array}{c}\text { Readmitted } \\
\text { Within } 30 \text { Days } \\
\text { of Index Admission }\end{array}$ & $\begin{array}{l}\text { Not Readmitted } \\
\text { Within } 30 \text { Days } \\
\text { of Index Admission }\end{array}$ & $p$-value & $\begin{array}{l}\text { Odds Ratio } \\
(95 \% \mathrm{Cl})\end{array}$ \\
\hline Substance Abuse (\%) & $2(10.0)$ & $21(8.3)$ & 0.68 & $1.22(0.27-5.63)$ \\
\hline Lack of Safety at Home (\%) & $16(80.0)$ & 99 (39.3) & 0.001 & $6.18(2.01-19.03)$ \\
\hline Anxiety $(\%)$ & $12(60.0)$ & $75(29.8)$ & 0.007 & $3.54(1.39-9.01)$ \\
\hline Medication Non-adherence (\%) & $7(35.0)$ & $45(17.9)$ & 0.08 & $2.48(0.93-6.56)$ \\
\hline Lack of Transportation (\%) & $7(35.0)$ & 39 (15.5) & 0.041 & $2.94(1.10-7.84)$ \\
\hline
\end{tabular}

multiple clinical and psychosocial comorbidities. We successfully demonstrated that those in the readmitted group had a higher rate of psychosocial risk factors such as anxiety, lack of transportation, and lack of home safety, compared to the non-readmitted group. However, to our surprise, the readmitted group was associated with increased incidence of pneumonia, but not with other clinical comorbidities, as had been described in previous studies. ${ }^{6,7}$ There was no statistically significant difference in mean $\mathrm{FEV}_{1}$ between the groups, suggesting that readmission was not a function of disease severity.

It is not surprising that patients who were readmitted for COPD had a higher rate of psychological risk factors. In 2016, Singh et al demonstrated that depression, anxiety, alcohol abuse, and drug abuse are associated with a higher COPD readmission rate in Medicare beneficiaries. ${ }^{19}$ However, very little so far 


\section{Figure 1. Monthly 30-Day Rates of Readmission for Acute Exacerbation of Chronic Obstructive Pulmonary Disease}

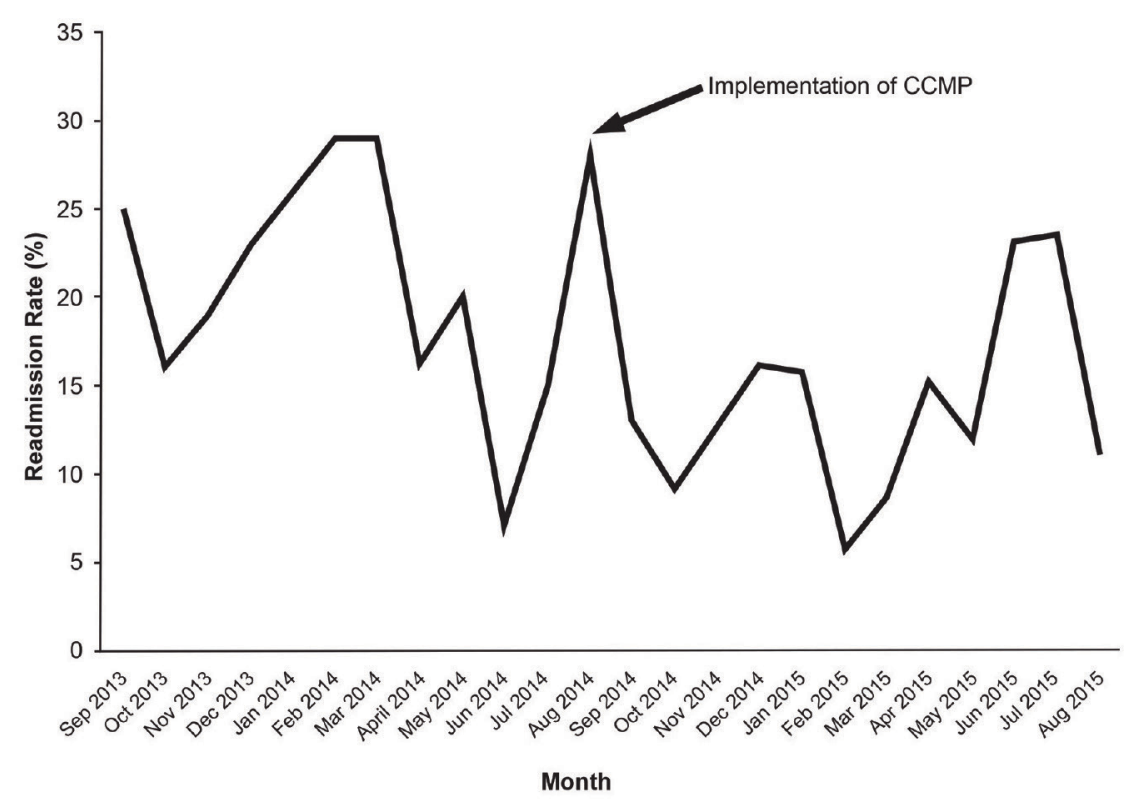

Monthly 30-day rates of readmission for acute exacerbation of chronic obstructive pulmonary disease during the 1-year periods pre- and post-implementation of a comprehensive care management program (CCMP) in September 2014. difference was not statistically significant $(p=0.075)$. Medication non-adherence has been demonstrated to be associated with increased mortality and hospital admissions in COPD. ${ }^{22,23}$ We suspect that this discrepancy might be caused by lack of power due to small sample size.

The implementation of our CCMP for patients with AECOPD was associated with a reduction in readmission rate by more than one-third. Interestingly, despite our success with a CCMP in reducing AECOPD readmissions, evidence regarding effectiveness of a CCMP for prevention of readmission after an AECOPD is controversial. In 2012, a randomized controlled trial conducted by Fan et al demonstrated that a COPD CCMP did not improve readmission rates in 20 Veterans Administration centers, and the study was terminated due to increased mortality in the CCMP group. ${ }^{12}$ In 2014 , in a parallelgroup, stratified, randomized controlled trial, Linden et al also failed to show an improvement in COPD readmission rates has been done to address anxiety in the formulation of CCMPs. Our study has also shown that home safety issues are associated with COPD readmissions. We speculate that this finding is explained by decreased activities of daily living performance in patients with COPD, as shown by a cross sectional study by Bendixen et al in $2014 .^{20}$ Living alone, lack of adequate support systems, and an unsafe structural environment puts these patients at risk for falls and increased shortness of breath due to exertion. Lack of security due to inadequate social support might also contribute to increased likelihood of seeking emergency medical care for minimal symptoms, as opposed to contacting their primary care providers or pulmonologists. Lack of transportation is a barrier to early outpatient intervention, which may contribute to more inpatient-based care for patients affected by this issue. In a systematic review in 2011, Keating et al demonstrated that lack of transportation affects use of pulmonary rehabilitation services, which are important for patients with COPD. ${ }^{21}$ Interestingly, although our study shows an ostensible difference in readmissions in patients who had difficulties in medication adherence (35.0\% versus $17.9 \%$ ), this in 2 community hospitals. ${ }^{13}$ However, as suggested by the authors, this study might have been limited by difference in intensity and duration of their education program, poor execution, lack of involvement of outpatient providers and availability of home visits. $^{14,15}$

Our COPD initiative differed from the CCMP in other studies in several aspects. First, our program strongly suggested a consultation with a pulmonologist for all admissions for AECOPD. We believe that involvement of pulmonologists within 24 hours of inpatient admission helped identify patients with high clinical risk and allowed for early intervention, optimization, and individualization of their care plan. Moreover, having multiple encounters with their consultants during their hospitalization might have led to a better patient-physician relationship, increasing patients' compliance. The important transition to outpatient care in our CCMP was facilitated by direct communication between an inpatient and outpatient care navigator, allowing for better transmission of clinical information to the outpatient setting. Furthermore, a modification of outpatient office workflow made space for early followups (within 5 days, often within 48 hours) as well as 
same day follow-ups (if a problem was identified), often solving challenges faced by patients after discharge without resorting to the emergency department. Using the outpatient care navigator to actively reach out to patients by phone and to receive calls also helped to identify patients in trouble, making possible the direct early intervention. We believe that the early involvement of a specialty service, the regimented use of a direct inpatient to outpatient transition of care and a flexible office schedule for same-day visits were the key to the successful decrease in AECOPD readmissions by our CCMP.

There are a few limitations to our study. First, as a single center study, selection bias can be a concern. However, as our study included almost all patients admitted to our hospital with the diagnosis of AECOPD, we believe this bias is mitigated and that our sample is a good representation of the general population treated in an academic community hospital. Second, the small sample size in the readmission group might have limited our power to show statistical significance in risk factors. However, despite the limited number of readmissions, we were able to show significant differences between patients who were readmitted and those who were not. The sample size may be masking differences such as medication nonadherence, which has been shown to be significant in other studies but had a $p$-value of 0.08 in our study. As a retrospective analysis, our design is subject to confounding factors and is not able to determine causation, but only association. Additionally, we were unable to determine whether any patients included in this study were subsequently admitted to another hospital. This information is relevant, as overall patient care would be improved only if patients refrain from admission to any hospital, not just ours. Of note, CMS would still penalize the index hospital for subsequent readmissions, regardless of hospital of readmission. Further investigation with a prospective cohort study and randomized controlled trial will be needed to further delineate the risk factors for readmissions and completeness of readmission data.

Results from our hospital and previous studies have suggested that there might be benefit in a COPD CCMP. From a community hospital that has successfully reduced readmissions for AECOPD after implementation of our CCMP, we speculate that a CCMP can reduce readmission rates with careful execution of a program that includes optimization of in-hospital care, better coordination of pre- and postdischarge care, attention to social variables, a system to better identify patient problems after discharge, and an office setup that can accommodate same day sick visits.

\section{Acknowledgements}

Author contributions: Drs. Wing Kong and Glenda Euceda were responsible for data collection and writing the article. Patricia Dilauro was involved in the initial data collection. John Chronakos was involved in the conception of the idea, data collection/analysis and revision of the article. Amber Butler was involved in the data collection and analysis and revision of the article. Rahila Ogunnaike was involved in the data collection and revision of the paper. All authors have reviewed and agree with the final manuscript.

\section{Declaration of Interest}

None of the authors have any financial, consulting, or personal relationships with people or organizations that might influence this work. 


\section{References}

1. Guarascio AJ, Ray SM, Finch CK, Self TH. The clinical and economic burden of chronic obstructive pulmonary disease in the USA. Clinicoecon Outcomes Res. 2013;5:235-245. doi: https://doi.org/10.2147/CEOR.S34321

2. Wier LM, Elixhauser A, Pfuntner A, Au DH. Overview of hospitalizations among patients with COPD, 2008: Statistical Brief \# 106. Healthcare Cost and Utilization Project Statistical Briefs: Rockville, MD; 2006.

doi: https://doi.org/10.2147/COPD.S51507

3. Baker CL, Zou KH, Su J. Risk assessment of readmissions following an initial COPD-related hospitalization. Int J Chron Obstruct Pulmon Dis. 2013;8:551-559.

4. Coventry PA, Gemmell I, Todd CJ. Psychosocial risk factors for hospital readmission in COPD patients on early discharge services: a cohort study. BMC Pulm Med. 2011;11:49-2466-11-49. doi: https://doi.org/10.1186/1471-2466-11-49

5. Park SK, Richardson CR, Holleman RG, Larson JL. Frailty in people with COPD, using the National Health and Nutrition Evaluation Survey dataset (2003-2006). Heart Lung. 2013; 42(3):163-170. doi: https://doi.org/10.1016/j.hrtlng.2012.07.004

6. Anecchino C, Rossi E, Fanizza C, et al. Prevalence of chronic obstructive pulmonary disease and pattern of comorbidities in a general population. Int $J$ Chron Obstruct Pulmon Dis. 2007;2(4):567-574.

7. Holguin F, Folch E, Redd SC, Mannino DM. Comorbidity and mortality in COPD-related hospitalizations in the United States, 1979 to 2001. Chest. 2005;128(4):2005-2011. doi: https://doi.org/10.1378/chest.128.4.2005

8. Shah T, Churpek MM, Coca Perraillon M, Konetzka RT. Understanding why patients with COPD get readmitted: a large national study to delineate the Medicare population for the readmissions penalty expansion. Chest. 2015;147(5):1219-1226. doi: https://doi.org/10.1378/chest.14-2181

9. Shah T, Press VG, Huisingh-Scheetz M, White SR. COPD readmissions: addressing COPD in the era of value-based health care. Chest. 2016;150(4):916-926.

doi: https://doi.org/10.1016/j.chest.2016.05.002

10. Braman SS. Hospital readmissions for COPD: we can meet the challenge. Chron Obstr Pulm Dis. 2015;2(1):4-7.

doi: http://doi.org/10.15326/jcopdf.2.1.2015.0130

11. Kruis AL, Smidt N, Assendelft WJ, et al. Integrated disease management interventions for patients with chronic obstructive pulmonary disease. Cochrane Database Syst Rev. 2013;(10):CD009437.

doi: https://doi.org/10.1002/14651858.CD009437.pub2

12. Fan VS, Gaziano JM, Lew R, et al. A comprehensive care management program to prevent chronic obstructive pulmonary disease hospitalizations: a randomized, controlled trial. Ann Intern Med. 2012;156(10):673-683.

doi: https://doi.org/10.7326/0003-4819-156-10-201205150-00003
13. Linden A, Butterworth S. A comprehensive hospital-based intervention to reduce readmissions for chronically ill patients: a randomized controlled trial. Am J Manag Care. 2014; 20(10):783792.

14. Bourbeau J, Julien M, Maltais F, et al. Reduction of hospital utilization in patients with chronic obstructive pulmonary disease: a disease-specific self-management intervention. Arch Intern Med. 2003;163(5):585-591.

doi: https://doi.org/10.1001/archinte.163.5.585

15. Laverty AA, Elkin SL, Watt HC, et al. Impact of a COPD discharge care bundle on readmissions following admission with acute exacerbation: interrupted time series analysis. PLoS One. 2015;10(2):e0116187.

doi: https://doi.org/10.1371/journal.pone.0116187

16. Adamson SL, Burns J, Camp PG, Sin DD, van Eeden SF. Impact of individualized care on readmissions after a hospitalization for acute exacerbation of COPD. Int J Chron Obstruct Pulmon Dis. 2016;11(1):61-71. doi: https://doi.org/10.2147/COPD.S93322

17. Global Initiative for Chronic Obstructive Lung Disease (GOLD). Global strategy for the diagnosis, management and prevention of COPD, 2017. GOLD website. http://goldcopd.org/gold-2017global-strategy-diagnosis-management-prevention-copd/ Published 2017. Accessed May 2018.

18. Macdonald MT, Lang A, Storch J, Stevenson L, Barber T, Iaboni $\mathrm{K}$, et al. Examining markers of safety in homecare using the international classification for patient safety. BMC Health Serv Res. 2013;13:191.

doi: https://doi.org/10.1186/1472-6963-13-191

19. Singh G, Zhang W, Kuo YF, Sharma G. Association of psychological disorders with 30-day readmission rates in patients with COPD. Chest. 2016;149(4):905-915. doi: https://doi.org/10.1378/chest.15-0449

20. Bendixen HJ, Waehrens EE, Wilcke JT, Sorensen LV. Selfreported quality of ADL task performance among patients with COPD exacerbations. Scand J Occup Ther. 2014;21(4):313-320. doi: https://doi.org/10.3109/11038128.2014.899621

21. Keating A, Lee A, Holland AE. What prevents people with chronic obstructive pulmonary disease from attending pulmonary rehabilitation? A systematic review. Chron Respir Dis. 2011;8(2):89-99. doi: https://doi.org/10.1177/1479972310393756

22. Vestbo J, Anderson JA, Calverley PM, et al. Adherence to inhaled therapy, mortality and hospital admission in COPD. Thorax. 2009;64(11):939-943.

doi: https://doi.org/10.1136/thx.2009.113662

23. van Boven JF, Chavannes NH, van der Molen T, Rutten-van Molken MP, Postma MJ, Vegter S. Clinical and economic impact of non-adherence in COPD: a systematic review. Respir Med. 2014;108(1):103-113. doi: https://doi.org/10.1016/j.rmed.2013.08.044 